\title{
Simulation-based design of vehicles exposed to blast threats for improved occupant survivability
}

\author{
R. T. Bocchieri, S. W. Kirkpatrick \& B. Peterson \\ Applied Research Associates, Inc., USA
}

\begin{abstract}
Designing vehicles to protect occupants from explosive threats is complicated by the complex set of physics that occurs from the point of detonation to the response of the occupants. These physics include detonation chemistry, shock physics, solid mechanics, structural dynamics, nonlinear material behaviour, and human physiology and injury mechanics, among others. Consequently, vehicles are typically developed through an iterative process of destructive field testing in order to determine the level of blast protection. This testing is both time consuming and costly and does not ensure an optimized design.

Recent advances in computational power and high-fidelity multi-physics computational tools now offer the alternative of performing Simulation-Based Design (SBD), similar to what is currently done for crash protection. Here, the explosive threat, its coupling to a vehicle, the vehicle structure, occupants or surrogates, and their coupling to the vehicle are all modelled in a single analysis. In this paper we describe an approach that applies state-of-the-art nonlinear finite element analysis to rapidly determine the survivability of vehicle designs and occupant injury potential when subjected to a buried charge or an above-ground improvised explosive device (IED). This integrated analysis capability allows iterative assessments to be performed as part of the design process. Modelling approaches for buried charges and IEDs and the coupling of the resulting loads on the vehicle are discussed. Finally, detailed sub-modelling of critical components such as occupant seating systems is demonstrated.
\end{abstract}

Keywords: armoured vehicle, blast, mine, IED, occupant survivability. 


\section{Introduction}

Mine blasts and improvised explosive devices (IEDs) pose a serious threat to occupants of armoured vehicles. These threats result in a variety of insults to occupants: fire, fragment penetration, primary blast loading, and both global and local blast-induced vehicle motion. Vehicles must be able to reasonably withstand these threats in order to maintain crew survivability. Of these threats, designing vehicles to be more survivable against blast-induced global vehicle kinematic motion and high-rate local deformation poses a significant challenge. Improved armour solutions can reduce injuries from projectiles, for example, however they can also result in greater injuries from blunt-trauma related vehicle motions. These types of design tradeoffs interact at a vehicle system level.

During vehicle development, contractors typically provide prototype vehicles for destructive field testing in order to determine the level of blast protection. This testing is both time consuming and costly and does not ensure an optimized design. A variety of field conditions and other related factors tend to make each blast test unique to some degree. Researchers struggle with comparative testing of candidate blast protection articles, and are usually left to rely on field experience and intuition when developing and finalizing protective designs.

Developing a virtual environment to evaluate competing protective designs is crucial. This environment would allow the designer to analyze and evaluate a large array of test conditions and prototypes. It would also offer a first level optimization of protective blast protection designs prior to committing to destructive field testing. This design methodology would significantly reduce development costs and produce a better vehicle.

\section{Simulation-based design}

The concept of a Simulation-Based Design (SBD) system is to apply numerical simulation methods that allow for the rapid iterative evaluation of various concepts in the design process. Such a system would allow the user to manipulate features of the design and get rapid feedback on the effects of changing these features. Features that a designer may wish to manipulate include vehicle geometry, material properties, material thicknesses, and threat types and locations. The feedback to the user may include various evaluation criteria such as damage to the vehicle or injury to human occupants. Ideally, a SBD system would offer a convenient interface, run quickly, and easily incorporate new vehicles, technologies, designs, or threats.

A simplified flow chart illustrating the main components of an SBD system is shown in Figure 1. Good engineering software tools already exist for many portions of the system for many applications. In many cases, development is needed to link them together in an automated fashion. A Graphical User Interface (GUI) would ideally be used to provide easy-to-use input menus. A computer-aided design (CAD) system is used to create the geometry of the entities to be modelled and automeshing software then converts the CAD description of the geometry into a computational mesh. At the core of this 
methodology is a physics-based solver (e.g., finite element (FE) code, Computational Fluid Dynamics (CFD) code) suitable for modelling the physical behaviour. Pre-determined performance measures are then extracted from the physical simulation and an optimizer selects the parameters for the next design iteration. The degree to which this process can be automated will depend on the complexity of the system to be designed.

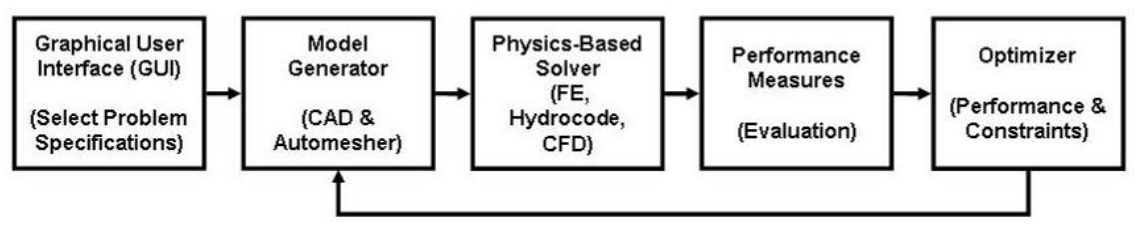

Figure 1: $\quad$ Simulation-based design flow chart.

Numerical modelling of the effects of blasts on vehicles involves modelling the blast source, coupling the loading from this source to the vehicle structure, and simulating the vehicle response and that of the occupants. Modelling methods range from empirical models of the mine blast and analytical models for simple vehicle structures to fully coupled simulations using multi-physics computational codes. Because of the demonstrated accuracy of these codes and the relatively cheap computational power in recent years, use of multi-physics codes can now be used as the primary solver for performing simulation-based design of armoured vehicles.

The vehicle blast demonstrations in this paper use LS-DYNA as the primary solver. LS-DYNA is a commercially available nonlinear explicit finite element code for the dynamic analysis of structures [1]. LS-DYNA is developed and supported by the Livermore Software Technology Corporation (LSTC) and is used for a wide variety of crash, blast, and impact applications. LS-DYNA has several unique capabilities for application to blast simulation of armoured vehicles. These included numerical techniques that enable modelling of the blast threats: Smooth Particle Hydrodynamics (SPH) and Arbitrary LagrangianEulerian (ALE).

\section{Modelling of threats}

Modelling of explosive threats is complex and high-fidelity simulations require a code that includes multi-physics capabilities. Important mechanisms to model for these threats include detonation physics and shock wave propagation, soil and or metallic casing mechanics and subsequent expansion of the detonation products and soil/fragment ejecta. Following are examples of modelling methods used for shallow-buried explosives and a representative IED.

\subsection{Shallow-buried explosives (mine blast)}

In order to develop and validate a generic mine threat model, an experiment performed by Defence R\&D Canada (DRDC) with an offset aluminium armour 
plate was selected [2]. In the experiment, a 6.3-kg bare explosive charge was buried in $5 \mathrm{~cm}$ of soil. A 3.175-cm thick aluminium (Al 5083) plate was placed on a support stand $40.64 \mathrm{~cm}$ from the top of the soil. A steel support and 10 metric tons of mass were placed on top of the aluminium plate to simulate proper vehicle weight. The LS-DYNA model developed for this test configuration is shown in Figure 2. The explosive charge and surrounding dry sand were modelled using SPH elements. The far field sand is modelled using Lagrangian elements.

The computed plate deformation is shown in Figure 3, and matches well with the published experimental results. The experiment showed a $30 \mathrm{~cm}$ displacement at the centre of the plate compared with $35 \mathrm{~cm}$ for the calculation. Both the experiment and the calculation showed a $5 \mathrm{~cm}$ edge displacement. Overall, the computed shape of the plate matches experimental observations.

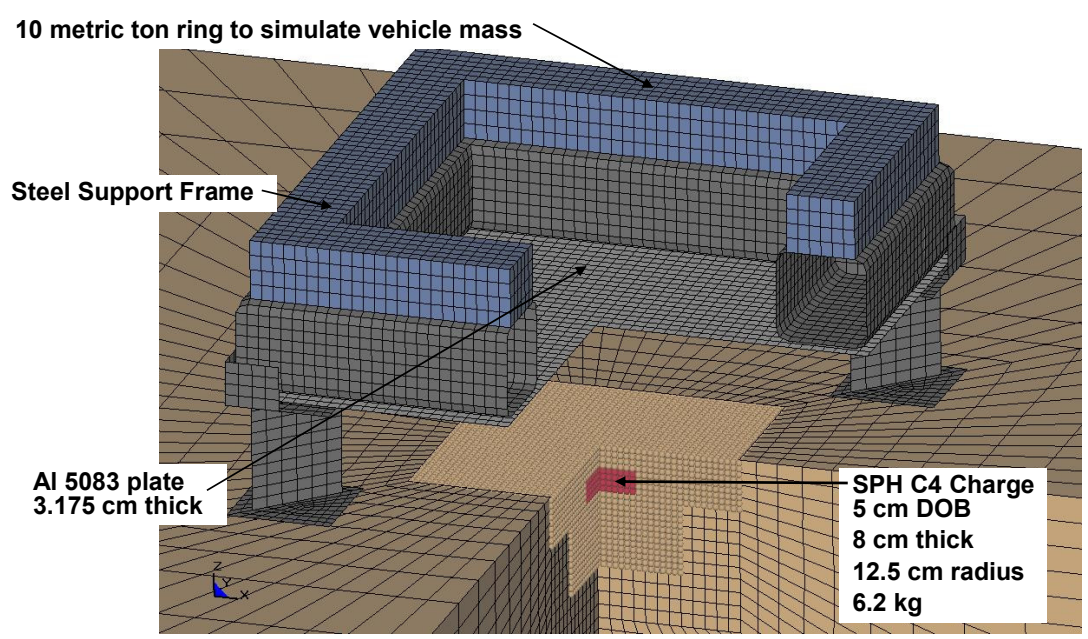

Figure 2: LS-DYNA geometry for the DRDC experiment.

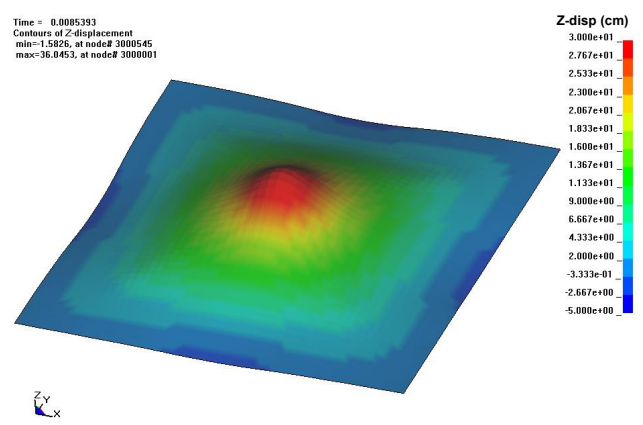

Figure 3: $\quad$ Plate deformation from LS-DYNA SPH calculation. 


\subsection{Improvised explosive device (IED)}

Cased explosive fragmenting weapons, such as an artillery shell, are commonly used in IEDs. Modelling such a weapon is complicated by the effects of the fragmenting case. The detonation of the explosive fill is initially confined in the case, and fragments the case as it expands. This fragmentation process develops during the detonation and initial expansion of the explosive products. Case material is rapidly expanded outward as the fragmentation progresses. For objects very close to the weapon, they are impacted by more intact components of the casing. The load on these objects more closely resembles the impact of a continuous material, rather than discrete fragments. At far distances, the fragmentation process has completed and the fragments have had time to spread out. Loading on far-field objects is best described as that from discrete fragment impacts.

Loading from the IED comes from significant contributions of both the airblast and fragment impacts. The pressure-time history and shape of this airblast wave front is dependent on the explosive weight, case geometry and material properties, and standoff distance. A simplified approach to modelling the effect of the case-fragmenting IED is shown in Figure 4. In this model, a Lagrangian mesh was used for the steel case and an ALE mesh was used for the high explosive (HE) fill inside the case as well as the surrounding air. An alternative modelling approach would be to use an ALE mesh for the case as well. For close-in applications, the ALE approach is appropriate as case fragmentation has not occurred.

An example simulation was performed of the IED interacting with a plate of Rolled Homogenous Armour (RHA), a material commonly used in armoured vehicles. Figure 5(a) shows the model geometry, where the steel plate is modelled with Lagrangian shell elements and the air, explosive, and steel case are modelled with ALE elements. The resulting damage to a one-inch and a onehalf-inch plate from detonation of the IED is shown in Figures 5(b) and 5(c), respectively. Such simulations are useful for validating threat models of IEDs using results from relatively inexpensive experiments.

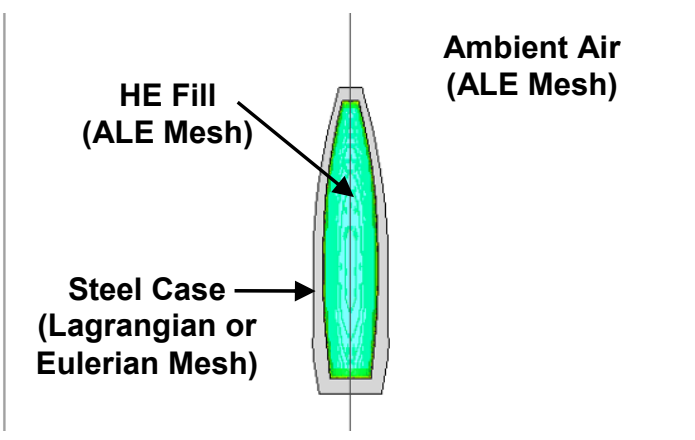

Figure 4: Model configuration for the IED detonation simulation. 


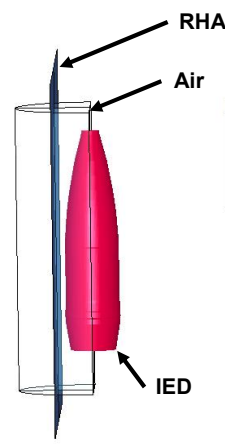

(a) Model Geometry

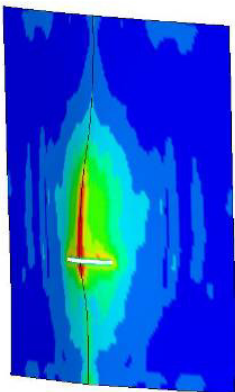

(b) One inch thick

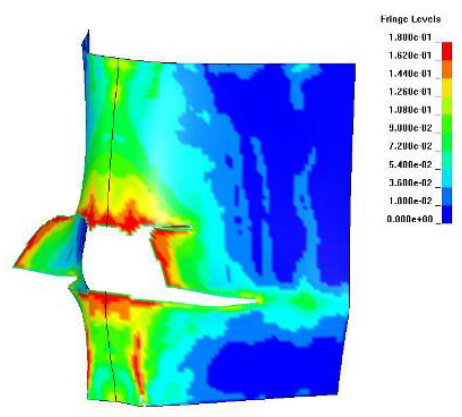

(c) One half inch thick

Figure 5: RHA plate deformations from near-field IED simulations. (Contours of effective plastic strain are shown.)

\section{Demonstrations of fully-coupled blast analyses of vehicles}

For many applications, coupling of loads to a structure can be accomplished using the traditional 'uncoupled' or 'loosely' coupled approach. With this approach, loading is applied from separate empirical model or multi-physics analysis of an explosive threat. Such an approach is appropriate when the structural response time is greater than the blast load duration. A typical application of this method is for airblast loads on most engineered building structures. However, this assumption may not hold when lighter or thinner structures such as window systems or vehicle components are considered and impacted by a mixed phase flow (such as air/soil). As the characteristic response time of the structures or components of interest approach the characteristic loading duration, the uncoupled approach can produce misleading results (e.g., [3]).

A fully-coupled analysis provides simultaneous solution of fluids, soil, casing and structural response for as much as every time step of a computational analysis. Such an analysis is needed when the structural response time is comparable or less than the blast load duration. A significant advantage of a fully-coupled analysis regardless of response time is that it provides the capability to perform a single end-to-end simulation of the event in one analysis. Mine blast has been shown to be one application where this approach can be needed for some vehicle structures. Two examples of fully-coupled analyses from the threat models discussed are summarized here.

\subsection{Mine blast beneath an armoured personnel carrier}

The first example of a fully-coupled analysis appropriate for SBD is that of an armoured personnel carrier with a land mine beneath a track. Positioning of the M113A3 on the explosive charge and ground model is shown in Figure 5. The vehicle is aligned so that the charge under the second road wheel on the left-hand 

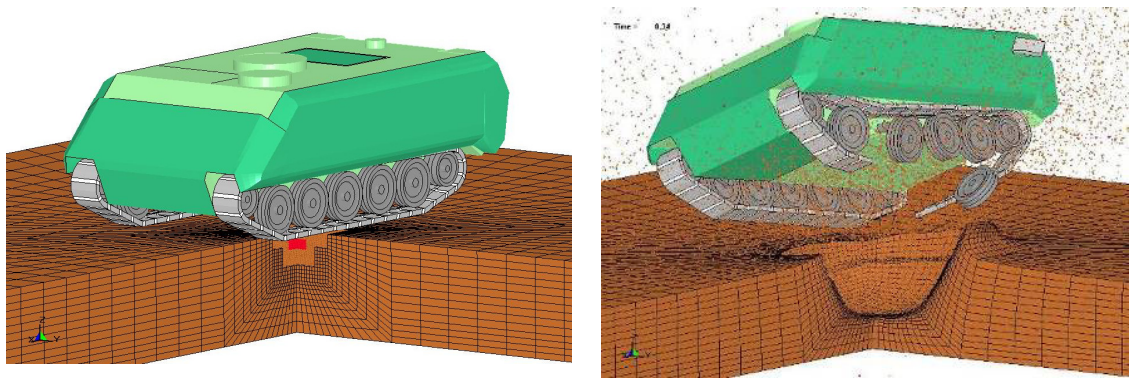

Figure 6: LS-DYNA SPH calculation of a buried bare charge against an M113A3.

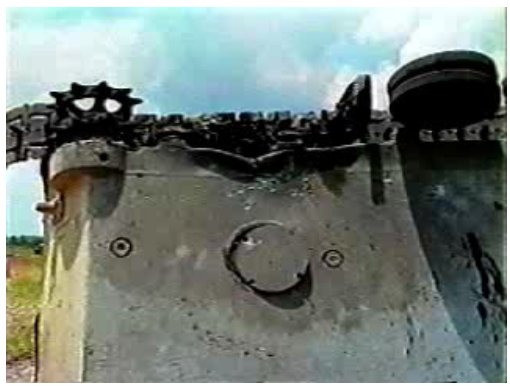

(a) Test

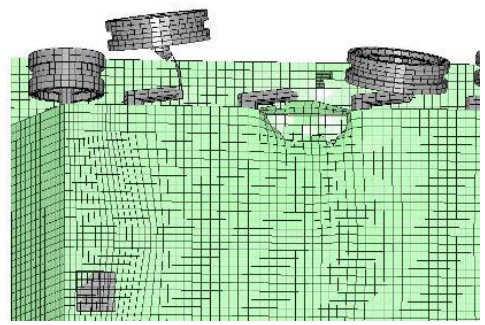

(b) Calculation

Figure 7: $\quad$ Comparison of tested and calculated damage to M113A3.

side of the vehicle. The charge zone, including the local area soil and charge, are modelled using SPH elements, which was previously described. Beyond the charge zone, the global soil area was modelled using Lagrangian elements.

A comparison of the calculated damage to the hull to a corresponding buried charge test is shown in Figure 6. The calculated damage matches the experiment quite well. In both the experiment and calculation the first two road wheels have failed, and there is a large deformation of the hull near the second road wheel. These calculations give an example of how a SBD system can be used to quickly analyze and compare different threats against a vehicle.

\subsection{Near-field IED simulation on an 'up-armoured' Chevrolet C2500 pickup truck}

The second example of a fully-coupled analysis appropriate for SBD uses the IED threat model developed against a notional up-armoured pickup truck for a near-field detonation. The vehicle modelled in this simulation was the uparmoured 1989 Chevrolet C2500 pickup truck. The pickup model was originally developed by the U.S. Federal Highway Administration (FHWA) for highway crashworthiness studies $[4,5]$. It has been notionally 'up-armoured' here to 
provide a more realistic representation of an armoured vehicle response to an IED or mine blast.

In order to reinforce the cab, the components shown in grey have been replaced with 0.4 inch RHA. A 1.0 inch thick plate of RHA, shown in tan, has been added under the cab in order to protect against mines and IEDs. To support the increased weight of this armour, the thicknesses of frame components, shown in red, have been doubled. The new total weight of the vehicle is 3.08 tons (a $62 \%$ increase from the un-armoured weight). Naturally, a complete model would require modifications to the suspension and possibly other components to support the added weight. Recall that the bottom armour plate thickness offers significant protection against this IED even at zero standoff.

A model of the IED was placed directly beneath the crew cab of the pickup and centred between the vehicle doors, as shown in Figure 8. The ALE mesh encompasses much of the vehicle cab. In this simulation, the IED explosive products and case material have been represented in the ALE mesh, while the pickup is comprised of Lagrangian shell elements. The IED has been placed on top of a rigid ground, best representing a surface such as concrete.
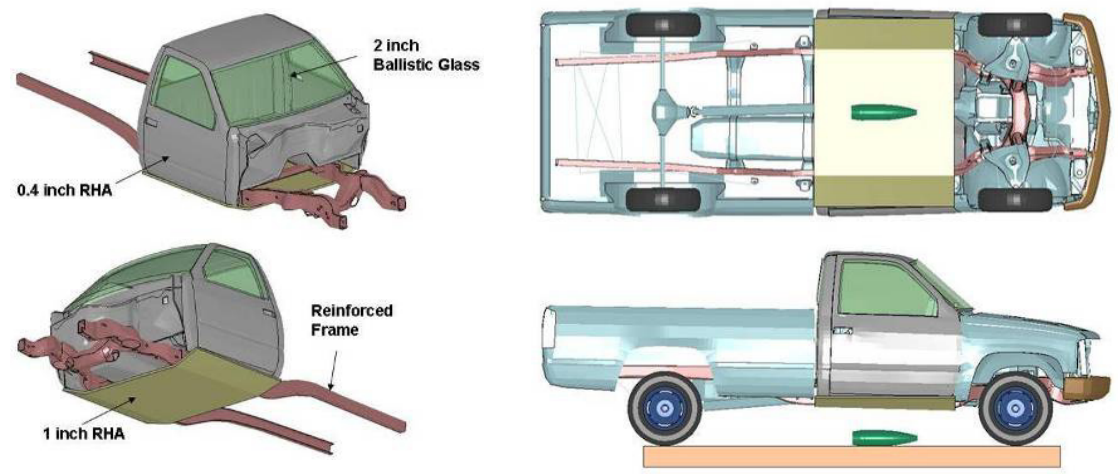

Figure 8: $\quad$ IED location beneath the up-armoured C2500 pickup truck.

The vehicle response to the IED detonation is shown in Figure 9 with contours of pressure superimposed on the model. The vehicle is viewed in the figure with a cutaway through the centreline of the pickup. In the simulation, the front of case material is completely stopped by the 1 inch plate of RHA. However, a pressure shock does propagate through the armour plate, through the cab floor, and into the occupant compartment. Associated damage to the vehicle is also shown in the figure with contours of plastic strain. There is significant plastic deformation in both the armour plate and the vehicle frame.

\section{Occupant injury assessment}

To ensure the survivability of occupants in armoured vehicles requires many forms of protection. Typically, armour is applied to provide protection against 
ballistic and blast threats. However, as the vehicles become lighter the same blast threats impart larger gross vehicle motions. An armour solution may be designed at the material-level to stop the ballistic threats, but the vehicle is still thrown so violently that the occupants are still injured or killed from the blunttrauma injuries induced by the severe acceleration environment. Because the response of an entire vehicle affects the accelerations of the occupants, including occupant models to assess injury potential in a SBD program is critical. Following is an example where computational models of Hybrid III dummies are used in a full vehicle analysis.

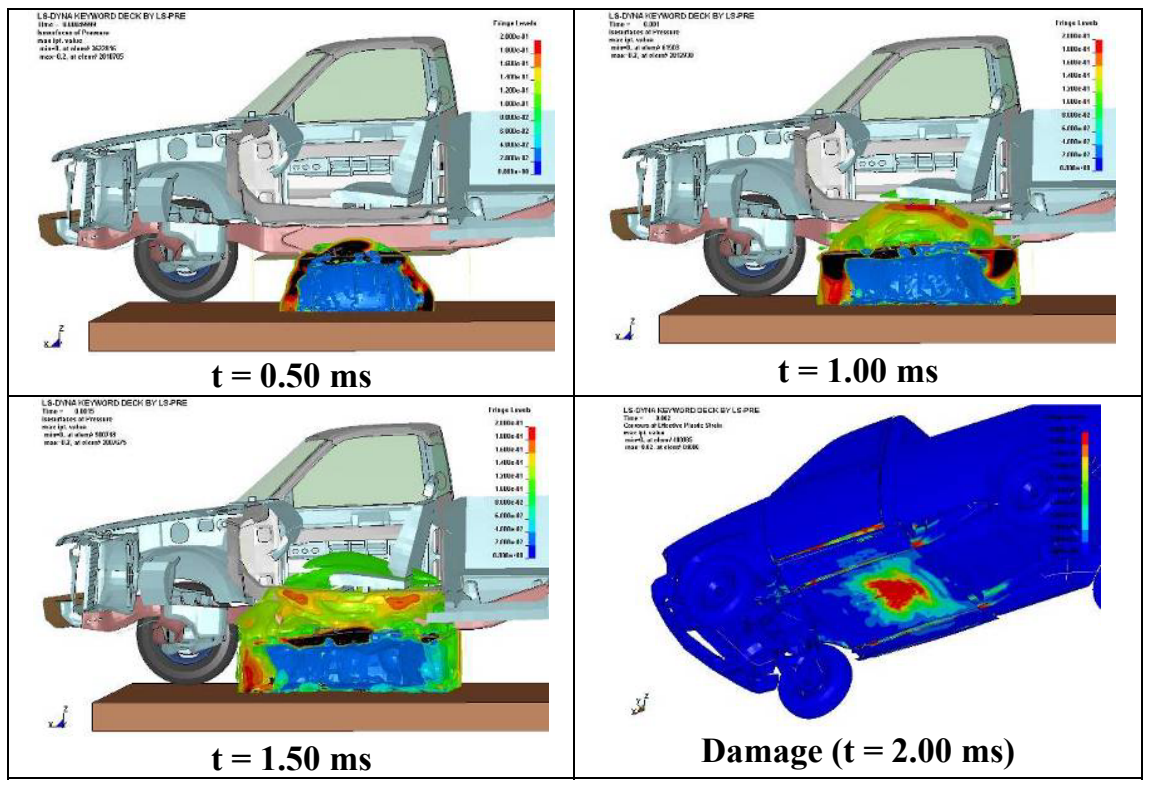

Figure 9: Pressure contours and damage from an IED detonation beneath the C2500 pickup truck.

Simulation of the M113A3 exposed to a mine blast discussed in the previous section included explicit modelling of six occupants placed in the passenger compartment of the vehicle [6]. Figure 10 shows the vehicle and occupant response at various times. At early times, the loading is transmitted through the floor to the lower extremities of the occupants. The subsequent loading results in uncontrolled occupant motions that are upward and out of their seated positions. From this occupant response, several injury criteria can be evaluated.

In this example, all of the occupants experienced a loading below the threshold Head Injury Criterion (HIC) value of 1000 (15 percent risk of an Abbreviated Injury Scale (AIS) 4+). The occupant closest to the mine (forward left position) had a HIC level of approximately 980. The head injury potential dropped rapidly for the other occupants. Subsequent motion of the unrestrained occupants also caused head strike injuries. The left middle occupant, for example resulted in a HIC level of approximately 700 . 


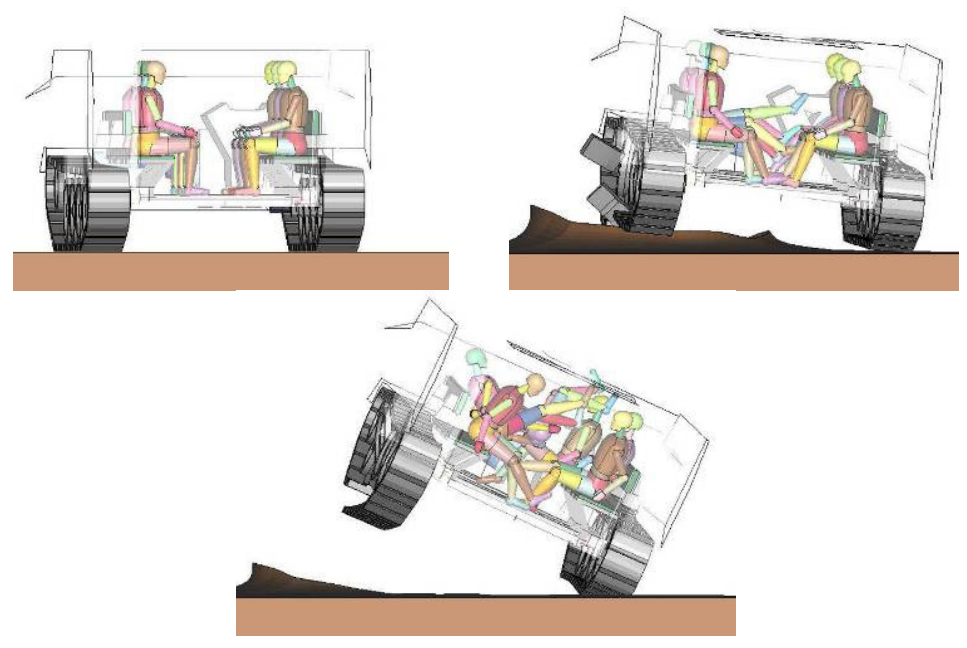

Figure 10: M113 mine blast simulation with occupants.

\section{SBD of seating systems using sub-modelling}

The SBD approach discussed thus far has been on the assessment of the entire vehicle in response to given threats. Full vehicle models were constructed with sufficient fidelity to assess the vehicle damage while maintaining reasonable run times. As a result, the models are not necessarily suitable for detailed aspects of the vehicle structure, such as detailed design of a shock-absorbing occupant seat system, or modelling of localized threats (e.g., penetration or an explosively formed projectile (EFP). Sub-model development allows higher fidelity simulations aspects without affecting the run times of the global vehicle model.

The use of sub-modelling during SBD is demonstrated here for a vehicle seating system. Seats frequently contain detailed components, such as energyabsorbing features, that are critical to the seat performance. Evaluations of these components required detailed modelling procedures that cannot be efficiently performed inside of global vehicle response simulations. In addition, the design process for the seating system is commonly performed independently from much of the vehicle design effort. Sub-modelling is a good approach to address these issues. This modelling approach is valid to assess the response of any critical equipment mounted to the vehicle interior.

In the first stage of the vehicle sub-modelling analysis, the seat design may not be know. But, it is important to include the occupants and seat in global simulations as they affect the vehicle response to the threat. For this purpose, an estimated low-fidelity model of a seating system is developed to incorporate in a full vehicle model in order to perform global simulations. Once the global simulations and vehicle design are complete, detailed design and modelling of the seating systems are then performed.

An example sub-modelling approach for the M113 and a prototype seat system is shown in Figure 11. In the global simulation, the response of the 
occupant crew compartment is extracted and saved. A simplified sub-model of the crew compartment is then constructed with detailed models of potential seat designs and the compartment response from the global analysis is applied. Modelling the entire compartment is important because the occupant response may involve interaction with surrounding vehicle structures and not just the seat structure.

With the faster run times of the sub-model, many design iterations are feasible. The seat can be modified and simulations performed with the new design. Once the final design has been determined the global model with the detailed component could be run to confirm the results.
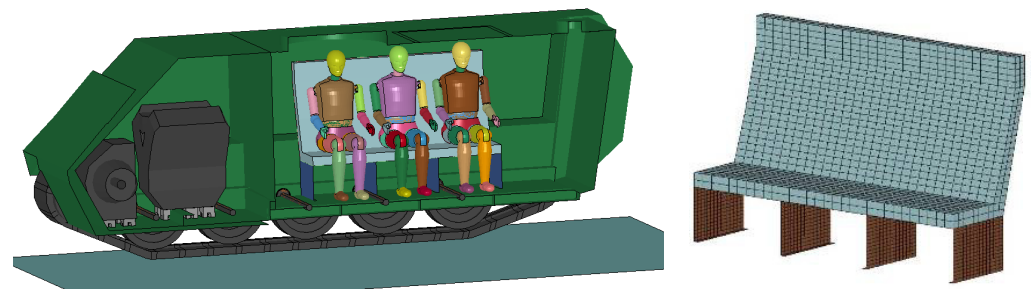

(a) Cutaway of M113 showing occupant compartment with simplified seat.
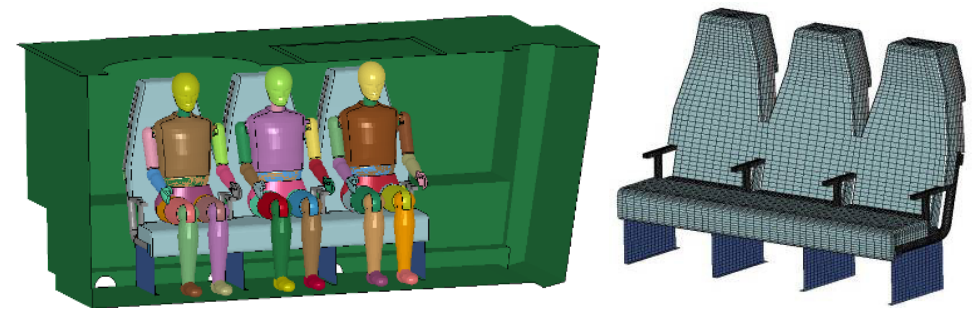

(b) Cutaway of the occupant compartment for sub-modelling with a detailed seat.

Figure 11: Cutaway views of global and sub-models of the M113.

\section{Conclusion}

A well-run SBD vehicle development program utilizes various types of software to automate the development of computational models, simulate response of the vehicle, extract performance metrics and optimize the design. The degree to which this process is automated depends on the complexity of the system to be designed. At the core of this methodology is a physics-based solver suitable for modelling the physical behaviour and the development of accurate threat, vehicle, and occupant models.

In order for the SBD approach to be successful, representative threats need to be defined carefully. Then models for these threats need to be developed and validated. Validation can be performed using simple experiments such as blast tests against flat plates or simple structures and with devices to measure the loads 
and load distributions from the threats. Vehicle models need to be developed with sufficient fidelity to simulate desired behaviours (armour failure, suspension behaviour, etc.). Component tests can be used to validate the model fidelity. Finally SBD trade studies can be conducted with threats at prescribed locations. Structural response metrics and occupant injury measures are extracted and compared against requirements. Once the initial investment of developing these models has been made, the cost savings of the SBD approach compared to destructive vehicle testing quickly becomes evident.

\section{Acknowledgement}

Support for this research by the U.S. Army RDECOM under the direction of Richard C. Goetz is gratefully acknowledged.

\section{References}

[1] "LS-DYNA Keyword User's Manual," Livermore Software Technology Corporation, Version 970, April 2003.

[2] Williams, K., "Validation of a Loading Model for Simulating Blast Mine Effects on Armoured Vehicles," 7th Int. LS-DYNA Users Conf., May 2002.

[3] Peterson B. D., S. W. Kirkpatrick, R. T. Bocchieri, "Advances in Finite Element Simulation of Blast Response to Structures", 48th AIAA/ASME/ASCE/AHS/ASC Structures, Structural Dynamics, and Materials Conf., Honolulu, Hawaii, 23-26 April, 2007.

[4] Tiso, P., C. Plaxico, M. Ray, and D. Marzougui, 'An Improved Truck Model for Roadside Safety Simulations: Part II - Suspension Modelling', Transportation Research Record No. 1797, 2002, p. 63-71.

[5] National Crash Analysis Centre Finite Element Model Archive, http://www.ncac.gwu.edu/vml/models.html.

[6] Kirkpatrick, S.W., MacNeill, R.A., and Bocchieri, R.T., "Development of an LS-DYNA Occupant Model for use in Crash Analyses of Roadside Safety Features," Transportation Review Board, No. TRB2003-0002450, Proc. of the 2003 TRB 82nd annual meeting, Washington D.C., Jan. 12-16, 2003. 